Proyecciones Journal of Mathematics

Vol. 34, No 2, pp. 175-190, June 2015.

Universidad Católica del Norte

Antofagasta - Chile

\title{
The largest Laplacian and adjacency indices of complete caterpillars of fixed diameter
}

\author{
Nair Abreu* \\ Universidade Federal do Ro de Janeiro, Brasil \\ Eber Lenes ${ }^{\dagger}$ \\ Universidad del Sinú, Colombia \\ and \\ Oscar Rojo \\ Universidad Católica del Norte, Chile \\ Received: January 2015. Accepted: May 2015
}

\begin{abstract}
A complete caterpillar is a caterpillar in which each internal vertex is a quasi-pendent vertex. In this paper, in the class of all complete caterpillars on $n$ vertices and diameter $d$, the caterpillar attaining the largest Laplacian index is determined. In addition, it is proved that this caterpillar also attains the largest adjacency index.
\end{abstract}

Keywords : Caterpillar, Laplacian matrix, Laplacian index, adjacency matrix, index, spectral radius.

2010 AMS classification : 05C50.

*thanks the support of Grant 305372/2009-2, CNPq, Brazil.

${ }^{\dagger}$ thanks the support of Project Mecesup UCN 0711 and Project Fondecyt Regular 1130135, Chile.

${ }^{\ddagger}$ thanks the support of Project Fondecyt Regular 1130135, Chile, and the hospitality of the Center For Mathematical Modeling, Universidad de Chile, Chile, in which this research was finished. 


\section{Introduction}

Let $G$ be a simple undirected graph on $n$ vertices. Let $D(G)$ be the diagonal matrix whose $(i, i)$-entry is the degree of the $i-t h$ vertex of $G$ and let $A(G)$ be the adjacency matrix of $G$. The matrix $L(G)=D(G)-A(G)$ is the Laplacian matrix of $G . L(G)$ is a positive semidefinite matrix and $(0, \mathbf{e})$ is an eigenpair of $L(G)$ where $\mathbf{e}$ is the all ones vector. The eigenvalues of $A(G)$ are called the eigenvalues of $G$ while the eigenvalues of $L(G)$ are called the Laplacian eigenvalues of $G$. The largest eigenvalue $\mu_{1}(G)$ of $L(G)$ is known as the Laplacian index of $G$ and the largest eigenvalue $\lambda_{1}(G)$ of $A(G)$ is the adjacency index or index of $G[1]$.

Let $\mathcal{T}_{n, d}$ be the class of all trees on $n$ vertices and diameter $d$. Let $P_{m}$ be a path on $m$ vertices and $K_{1, p}$ be a star on $p+1$ vertices.

In [9] the authors prove that the tree in $\mathcal{T}_{n, d}$ having the largest index is the caterpillar $P_{d, n-d}$ obtained from $P_{d+1}$ on the vertices $1,2, \ldots, d+1$ and the star $K_{1, n-d-1}$ identifying the root of $K_{1, n-d-1}$ with the vertex $\left\lceil\frac{d+1}{2}\right\rceil$ of $P_{d+1}$. In [2], for $3 \leq d \leq n-4$, the first $\left\lfloor\frac{d}{2}\right\rfloor+1$ indices of trees in $\mathcal{T}_{n, d}$ are determined. In [3], for $3 \leq d \leq n-3$, the first $\left\lfloor\frac{d}{2}\right\rfloor+1$ Laplacian spectral radii of trees in $\mathcal{T}_{n, d}$ are characterized.

In a graph a vertex of degree at least 2 is called an internal vertex, a vertex of degree 1 is a pendant vertex and any vertex adjacent to a pendant vertex is a quasi-pendant vertex. We recall that a caterpillar is a tree in which the removal of all pendant vertices and incident edges results in a path. We define a complete caterpillar as a caterpillar in which each internal vertex is a quasi-pendant vertex.

Let $d \geq 3, n>2(d-1)$ and $\mathbf{p}=\left[\begin{array}{llll}p_{1} & \ldots & \ldots & p_{d-1}\end{array}\right]$.

Let $\mathcal{C}_{n, d}$ be the class of all complete caterpillars on $n$ vertices and diameter $d$. A caterpillar $C(\mathbf{p})$ in $\mathcal{C}_{n, d}$ is obtained from the path $P_{d-1}$ and the stars $K_{1, p_{1}}, K_{1, p_{2}}, \ldots, K_{1, p_{d-1}}$ by identifying the root of $K_{1, p_{i}}$ with the $i-t h$ vertex of $P_{d-1}$ where $p_{1} \geq 1, p_{2} \geq 1, \ldots, p_{d-1} \geq 1$ and $p_{1}+\ldots+p_{d-1}=$ $n-d+1$. A special subclass of $\mathcal{C}_{n, d}$ is $\mathcal{A}_{n, d}=\left\{A_{1}, A_{2}, \ldots, A_{d-2}, A_{d-1}\right\}$ where $A_{k}=C(\mathbf{p}) \in \mathcal{C}_{n, d}$ with $p_{i}=1$ for $i \neq k$ and $p_{k}=n-2 d+3$. 
Example 1. $A_{4}=C\left(\begin{array}{lllll}1 & 1 & 1 & 5 & 1\end{array}\right)$ is the caterpillar

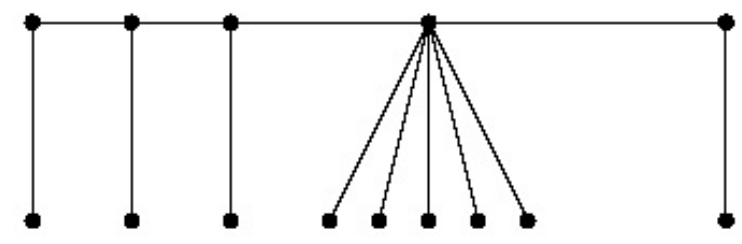

of 14 vertices and diameter 6 .

The complete caterpillars were initially studied in [5] and [6]. In particular, in [6] the authors determine the unique complete caterpillars that minimize and maximize the algebraic connectivity (second smallest Laplacian eigenvalue) among all complete caterpillars on $n$ vertices and diameter $d$. Below we summarize the result corresponding to the caterpillar attaining the largest algebraic connectivity.

Theorem 1. [6], Theorems 3.3 and 3.6. Among all caterpillars in $\mathcal{C}_{n, d}$ the largest algebraic connectivity is attained by the caterpillar $A_{\left\lfloor\frac{d}{2}\right\rfloor}$.

Numerical experiments suggest us that $A_{\left\lfloor\frac{d}{2}\right\rfloor}$ is also the caterpillar attaining the largest Laplacian index in the class $\mathcal{C}_{n, d}$. In this paper, we prove that this conjecture is true. Moreover, we prove that $A_{\left\lfloor\frac{d}{2}\right\rfloor}$ also attains the largest adjacency index in $\mathcal{C}_{n, d}$. To get these results, we first prove that the caterpillars in $\mathcal{C}_{n, d}$ attaining the mentioned largest indices lie in $\mathcal{A}_{n, d}$ and then we order the caterpillars in this subclass by their Laplacian indices as well as by their adjacency indices.

\section{The largest Laplacian index among all complete caterpil- lars}

Let $x_{1}, x_{2}, \ldots, x_{d-1}$ be the vertices of the path $P_{d-1}$ of the caterpillars $C(\mathbf{p}) \in$ $\mathcal{C}_{n, d}$. Let $C(\mathbf{p}) \in C_{n, d}$ with $\mathbf{p}=\left[p_{1}, p_{2}, \ldots, p_{d-1}\right]$. Then

$d\left(x_{1}\right)=p_{1}+1, d\left(p_{2}\right)=p_{2}+2, \ldots, d\left(x_{d-2}\right)=p_{d-2}+2, d\left(p_{d-1}\right)=p_{d-1}+1$.

Let $N_{G}(v)$ be the set of vertices in $G$ adjacent to the vertex $v$. 
Lemma 1. [3] Let $u, v$ be two vertices of a tree $T$. For $1 \leq s \leq d(v)$, let $v_{1}, v_{2}, \ldots, v_{s}$ be some vertices in $N_{T}(v)-\left(N_{T}(u) \cup\{u\}\right)$. For $1 \leq t \leq d(u)$, let $u_{1}, u_{2}, \ldots, u_{t}$ be some vertices in $N_{T}(u)-\left(N_{T}(v) \cup\{v\}\right)$. Let

$$
T_{u}=T-v v_{1}-v v_{2}-\cdots-v v_{s}+u v_{1}+u v_{2}+\cdots+u v_{s}
$$

and

$$
T_{v}=T-u u_{1}-u u_{2}-\cdots-u u_{t}+v u_{1}+v u_{2}+\cdots+v u_{t} .
$$

If both $T_{u}$ and $T_{v}$ are trees, then we have either $\mu_{1}\left(T_{u}\right)>\mu_{1}(T)$ or $\mu_{1}\left(T_{v}\right)>\mu_{1}(T)$.

We recall that $C(\mathbf{p})=A_{k} \in \mathcal{A}_{n, d}$ if and only if $p_{i}=1$ for $i \neq k$ and $p_{k}=n-2 d+3$.

Theorem 2. Let $d \geq 3$. Let $C(\mathbf{p}) \in \mathcal{C}_{n, d}$. Then there exists a caterpillar $A_{k} \in \mathcal{A}_{n, d}$ such that $\mu_{1}(C(\mathbf{p})) \leq \mu_{1}\left(A_{k}\right)$ for some $1 \leq k \leq d-1$.

Proof. Let $\# S$ be the cardinality of a set $S$. Let $d \geq 3$. Let $C(\mathbf{p}) \in \mathcal{C}_{n, d}$ with $\mathbf{p}=\left[\begin{array}{lllll}p_{1} & p_{2} & \ldots & \ldots & p_{d-1}\end{array}\right]$.

If $C(\mathbf{p}) \in \mathcal{A}_{n, d}$ then there is nothing to prove. Let $C(\mathbf{p}) \in \mathcal{C}_{n, d}-\mathcal{A}_{n, d}$. Let $S=\left\{1 \leq i \leq d-1: p_{i}>1\right\}$. Then $\# S \geq 2$. Let $i, j \in S$ with $i<j$. Let $u=x_{i}$ and $v=x_{j}$. Let $S(u)=\left\{u_{1}, u_{2}, \ldots, u_{p_{i}-1}, u_{p_{i}}\right\}$ and $S(v)=$ $\left\{v_{1}, v_{2}, \ldots, v_{p_{j}-1}, v_{p_{j}}\right\}$ be the sets of pendant vertices adjacent to $u$ and $v$, respectively. Let

$$
T_{u}=C(\mathbf{p})-v v_{1}-v v_{2}-\cdots-v v_{p_{j}-1}+u v_{1}+u v_{2}+\cdots+u v_{p_{j}-1}
$$

and

$$
T_{v}=C(\mathbf{p})-u u_{1}-u u_{2}-\cdots-u u_{p_{i}-1}+v u_{1}+v u_{2}+\cdots+v u_{p_{i}-1} .
$$

Then $T_{u}=C(\mathbf{q}) \in \mathcal{C}_{n, d}$ where $\mathbf{q}=\mathbf{p}$ except for $q_{i}=p_{i}+p_{j}-1$ and $q_{j}=1$ and $T_{v}=C(\mathbf{r}) \in \mathcal{C}_{n, d}$ where $\mathbf{r}=\mathbf{p}$ except for $r_{i}=1$ and $r_{j}=$ $p_{j}+p_{i}-1$. By Lemma $1, \mu_{1}\left(T_{u}\right)>\mu_{1}(C(\mathbf{p}))$ or $\mu_{1}\left(T_{v}\right)>\mu_{1}(C(\mathbf{p}))$. Suppose $\mu_{1}\left(T_{u}\right)>\mu_{1}(C(\mathbf{p}))$. Let $S_{1}=\left\{1 \leq i \leq d-1: q_{i}>1\right\}$. By the definition of $T_{u}, \# S_{1}=\# S-1$. Suppose now $\mu_{1}\left(T_{v}\right)>\mu_{1}(C(\mathbf{p}))$. Let $S_{2}=\left\{1 \leq i \leq d-1: r_{i}>1\right\}$. Also, by the definition of $T_{v}, \# S_{2}=\# S-1$. By a repeated application of the above argument, we finally arrive at a caterpillar $A_{k}=C(\widetilde{\mathbf{p}}) \in \mathcal{A}_{n, d}$ where $\widetilde{p}_{i}=1$ for all $i \neq k$ and $\widetilde{p}_{k}=n-2 d+3$ such that $\mu_{1}\left(A_{k}\right)>\mu_{1}(C(\mathbf{p}))$.

Corollary 1. If $d=3$ then $C(n-3,1)$ has the largest Laplacian index among all trees on $n$ vertices and diameter 3 . 
Proof. Since any tree $T$ on $n$ vertices and diameter 3 is a complete caterpillar, we may take $T=C\left(p_{1}, p_{2}\right) \in \mathcal{C}_{n, 3}$. By Theorem 2 , there exists $C_{1}=C\left(p_{1}+p_{2}-1,1\right)=C(n-3,1) \in \mathcal{C}_{n, 3}$ such that $\mu_{1}\left(C_{1}\right) \geq \mu_{1}(C)$ or there exists

$C_{2}=C\left(1, p_{1}+p_{2}-1\right)=C(1, n-3) \in \mathcal{C}_{n, 3}$ such that $\mu_{1}\left(C_{2}\right) \geq \mu_{1}(C)$. Since $C_{1}$ and $C_{2}$ are isomorphic caterpillars, the result follows.

From Theorem 2, it follows that among the caterpillars in $\mathcal{C}_{n, d}$ the largest Laplacian index is attained by a caterpillar in the subclass $\mathcal{A}_{n, d}$. Next, we order the caterpillars in $\mathcal{A}_{n, d}$ by their Laplacian indices.

A generalized Bethe tree is a rooted tree in which vertices at the same distance from the root have the same degree. In [7], we characterize the eigenvalues of the Laplacian and adjacency matrices of the tree $P_{m}\left\{B_{i}\right\}$ obtained from the path $P_{m}$ and the generalized Bethe trees $B_{1}, B_{2}, \ldots, B_{m}$ obtained by identifying the root vertex of $B_{i}$ with the $i-t h$ vertex of $P_{m}$. This is the case for $C(\mathbf{p})$ in which the path is $P_{d-1}$ and each star $K_{1, p_{i}}$ is a generalized Bethe tree of 2 levels. From Theorem 2 in [7], we get

Theorem 3. The Laplacian eigenvalues of $C(\mathbf{p})$ are 1 with multiplicity $\sum_{i=1}^{d-1} p_{i}-(d-1)$ and the eigenvalues of the $(2 d-2) \times(2 d-2)$ irreducible nonnegative matrix

$$
M(\mathbf{p})=\left[\begin{array}{ccccc}
T\left(p_{1}\right) & E & & & \\
E & S\left(p_{2}\right) & E & & \\
& \ddots & \ddots & \ddots & \\
& & \ddots & S\left(p_{d-2}\right) & E \\
& & & E & T\left(p_{d-1}\right)
\end{array}\right]
$$

where

$$
T(x)=\left[\begin{array}{cc}
1 & \sqrt{x} \\
\sqrt{x} & x+1
\end{array}\right], E=\left[\begin{array}{ll}
0 & 0 \\
0 & 1
\end{array}\right], S(x)=T(x)+E .
$$

Let $\rho(A)$ be the spectral radius of the matrix $A$.

Corollary 2. The matrix $M(\mathbf{p})$ is singular, $\rho(M(\mathbf{p}))>1$ and $\rho(M(\mathbf{p}))$ is the Laplacian index of $C(\mathbf{p})$. 
Proof. Since 0 is a Laplacian eigenvalue of any graph, an immediate consequence of Theorem 3 is that $M(\mathbf{p})$ is a singular matrix. Since $M(\mathbf{p})$ is a nonnegative irreducible matrix whose row sums are no constant, $\rho(M(\mathbf{p}))>1$ [10]. From this fact and Theorem 3, $\rho(M(\mathbf{p}))$ is the Laplacian index of $C(\mathbf{p})$.

Let $t(\lambda, x)$ and $s(\lambda, x)$ be the characteristic polynomials of the matrices $T(x)$ and $S(x)$ respectively. That is

$$
t(\lambda, x)=\lambda^{2}-(x+2) \lambda+1
$$

and

$$
s(\lambda, x)=\lambda^{2}-(x+3) \lambda+2 .
$$

Then

$$
s(\lambda, x)-t(\lambda, x)=1-\lambda .
$$

Let us denote by $|A|$ the determinant of a square matrix $A$ and by $\widetilde{B}$ the matrix obtained from a matrix $B$ by deleting its last row and its last column. We recall Lemma 2.2 in [8].

Lemma 2. For $i=1,2, \ldots, r$, let $B_{i}$ be a matrix of order $k_{i} \times k_{i}$ and $\mu_{i, j}$ be arbitrary scalars. Then

$$
\begin{gathered}
\left|\begin{array}{ccccc}
B_{1} & \mu_{1,2} E_{1,2} & \cdots & \mu_{1, r-1} E_{1, r-1} & \mu_{1, r} E_{1, r} \\
\mu_{2,1} E_{1,2}^{T} & B_{2} & \cdots & \cdots & \mu_{2, r} E_{2, r} \\
\mu_{3,1} E_{1,3}^{T} & \mu_{3,2} E_{2,3}^{T} & \ddots & \cdots & \vdots \\
\vdots & \vdots & \vdots & B_{r-1} & \mu_{r-1, r} E_{r-1, r} \\
\mu_{r, 1} E_{1, r}^{T} & \mu_{r, 2} E_{2, r}^{T} & \cdots & \mu_{r, r-1} E_{r-1, r}^{T} & B_{r}
\end{array}\right| \\
=\left|\begin{array}{cccc|c}
\left|B_{1}\right| & \mu_{1,2}\left|\widetilde{B_{2}}\right| & \cdots & \mu_{1, r-1}\left|\widetilde{B_{r-1}}\right| & \mu_{1, r}\left|\widetilde{B_{r}}\right| \\
\mu_{2,1}\left|\widetilde{B_{1}}\right| & \left|B_{2}\right| & \cdots & \cdots & \mu_{2, r}\left|\widetilde{B_{r}}\right| \\
\mu_{3,1}\left|\widetilde{B_{1}}\right| & \mu_{3,2}\left|\widetilde{B_{2}}\right| & \ddots & \cdots & \vdots \\
\vdots & \vdots & \vdots & \left|B_{r-1}\right| & \mu_{r-1, r}\left|\widetilde{B_{r}}\right| \\
\mu_{r, 1}\left|\widetilde{B_{1}}\right| & \mu_{r, 2}\left|\widetilde{B_{2}}\right| & \cdots & \mu_{r, r-1}\left|\widetilde{B_{r-1}}\right| & \left|B_{r}\right|
\end{array}\right| .
\end{gathered}
$$

The notation $|A|_{l}$ will be used to denote the determinant of the matrix $A$ of order $l \times l$.

The next result is an immediate consequence of the application of Lemma 2 to the characteristic polynomial of $M(\mathbf{p})$. 
Corollary 3. The characteristic polynomial of $M(\mathbf{p})$ is

$$
|\lambda I-M(\mathbf{p})|=\left|\begin{array}{ccccc}
t\left(\lambda, p_{1}\right) & 1-\lambda & & & \\
1-\lambda & s\left(\lambda, p_{2}\right) & 1-\lambda & & \\
& \ddots & \ddots & \ddots & \\
& & \ddots & s\left(\lambda, p_{d-2}\right) & 1-\lambda \\
& & & 1-\lambda & t\left(\lambda, p_{d-1}\right)
\end{array}\right|_{d-1} .
$$

From now on, let $a=n-2 d+3$ and let $\mathbf{a}_{k}$ be the $(d-1)-$ dimensional vector in which the $k$-th component is equal to $a$ and all the other components are equal to 1 . Using this notation, $A_{k}=C\left(\mathbf{a}_{k}\right)$. Since the Laplacian index of $C(\mathbf{p}) \in \mathcal{C}_{n, d}$ is the spectral radius of $M(\mathbf{p})$, to find an order in $\mathcal{A}_{n, d}$ by the Laplacian index is equivalent to order the matrices $M\left(\mathbf{a}_{1}\right), M\left(\mathbf{a}_{2}\right), \ldots, M\left(\mathbf{a}_{d-1}\right)$ by their spectral radii. Since $A_{k}$ and $A_{d-k}$ are isomorphic, we may take $1 \leq k \leq\left\lfloor\frac{d}{2}\right\rfloor$. Let $\phi_{k}(\lambda)$ be the characteristic polynomial of $M\left(\mathbf{a}_{k}\right)$, that is,

$$
\phi_{k}(\lambda)=\left|\lambda I-M\left(\mathbf{a}_{k}\right)\right| .
$$

By Corollary 3, the $(k, k)$ - entry of $\phi_{k}(\lambda)=\left|\lambda I-M\left(\mathbf{a}_{k}\right)\right|$ is $t(\lambda, a)$ if $k=1$ and $s(\lambda, a)$ if $k \neq 1$.

Let $\mathbf{e}_{l}$ be the all ones column vector with $l$ entries. Let $\varphi_{l}(\lambda)=$ $\left|\lambda I-M\left(\mathbf{e}_{l}\right)\right|$. By application of Corollary 3, we have

$$
\varphi_{l}(\lambda)=\left|\begin{array}{ccccc}
t(\lambda, 1) & 1-\lambda & & & \\
1-\lambda & s(\lambda, 1) & 1-\lambda & & \\
& \ddots & \ddots & \ddots & \\
& & \ddots & s(\lambda, 1) & 1-\lambda \\
& & & 1-\lambda & t(\lambda, 1)
\end{array}\right|_{l}
$$

Let

$$
r_{0}(\lambda)=1, r_{1}(\lambda)=t(\lambda, 1)
$$

and, for $2 \leq k \leq\left\lfloor\frac{d}{2}\right\rfloor$, let

$$
r_{k}(\lambda)=\left|\begin{array}{ccccc}
s(\lambda, 1) & 1-\lambda & & & \\
1-\lambda & \ddots & 1-\lambda & & \\
& \ddots & \ddots & \ddots & \\
& & \ddots & s(\lambda, 1) & 1-\lambda \\
& & & 1-\lambda & t(\lambda, 1)
\end{array}\right|_{k}
$$


Expanding along the first row, we obtain

$$
r_{k}(\lambda)=s(\lambda, 1) r_{k-1}(\lambda)-(\lambda-1)^{2} r_{k-2}(\lambda) .
$$

Since $s(\lambda, x)=t(\lambda, x)+1-\lambda$, by linearity on the first column, we have

$$
r_{k}(\lambda)=\left|\begin{array}{ccccc}
t(\lambda, 1) & 1-\lambda & & & \\
1-\lambda & s(\lambda, 1) & 1-\lambda & & \\
& \ddots & \ddots & \ddots & \\
& & \ddots & s(\lambda, 1) & 1-\lambda \\
& & & 1-\lambda & t(\lambda, 1)
\end{array}\right|_{k}+(1-\lambda) r_{k-1}(\lambda) .
$$

Therefore

$$
r_{k}(\lambda)=\varphi_{k}(\lambda)+(1-\lambda) r_{k-1}(\lambda) .
$$

Let $1 \leq k \leq\left\lfloor\frac{d}{2}\right\rfloor-1$. We search for the difference $\phi_{k}(\lambda)-\phi_{k+1}(\lambda)$. We recall that $(k, k)$ - entry of $\phi_{k}(\lambda)=\left|\lambda I-M\left(\mathbf{a}_{k}\right)\right|$ is $t(\lambda, a)$ if $k=1$ and $s(\lambda, a)$ if $k \neq 1$. Since $t(\lambda, a)=t(\lambda, 1)+(1-a) \lambda$ and $s(\lambda, a)=$ $s(\lambda, 1)+(1-a) \lambda$, by linearity on the $k-t h$ column, we have

$$
\begin{aligned}
& 3) \phi_{k}(\lambda)=\left|\begin{array}{ccccc}
t(\lambda, 1) & 1-\lambda & & & \\
1-\lambda & s(\lambda, 1) & 1-\lambda & & \\
& \ddots & \ddots & \ddots & \\
& & \ddots & s(\lambda, 1) & 1-\lambda \\
& & & 1-\lambda & t(\lambda, 1)
\end{array}\right|_{d-1} \\
& +(1-a) \lambda\left|\begin{array}{cc}
r_{k-1}(\lambda) \\
0 & 0 \\
&
\end{array}\right|
\end{aligned}
$$

The $(k+1, k+1)$-entry of the determinant of order $d-1$ on the second right hand of $(2.3)$ is $s(\lambda, 1)$ and since $s(\lambda, 1)=s(\lambda, a)+(a-1) \lambda$, by linearity on the $(k+1)-t h$ column, we obtain

$$
\left|\begin{array}{ccccc}
t(\lambda, 1) & 1-\lambda & & & \\
1-\lambda & s(\lambda, 1) & 1-\lambda & & \\
& \ddots & \ddots & \ddots & \\
& & \ddots & s(\lambda, 1) & 1-\lambda \\
& & & 1-\lambda & t(\lambda, 1)
\end{array}\right|_{d-1}
$$




$$
=\phi_{k+1}(\lambda)+(a-1) \lambda\left|\begin{array}{cc}
r_{k}(\lambda) & 0 \\
0 & r_{d-k-2}(\lambda)
\end{array}\right| .
$$

Replacing in (2.3), we get

$$
\begin{aligned}
& \phi_{k}(\lambda)-\phi_{k+1}(\lambda) \\
= & (1-a) \lambda\left|\begin{array}{cc}
r_{k-1}(\lambda) & 0 \\
0 & r_{d-k-1}(\lambda)
\end{array}\right|+(a-1) \lambda\left|\begin{array}{cc}
r_{k}(\lambda) & 0 \\
0 & r_{d-k-2}(\lambda)
\end{array}\right| .
\end{aligned}
$$

Thus

$$
\phi_{k}(\lambda)-\phi_{k+1}(\lambda)=(a-1) \lambda\left[r_{k}(\lambda) r_{d-k-2}(\lambda)-r_{k-1}(\lambda) r_{d-k-1}(\lambda)\right] .
$$

Applying the recurrence formula (2.1) to $r_{k}(\lambda)$ and $r_{d-k-1}(\lambda)$, we obtain

$$
\begin{aligned}
& r_{k}(\lambda) r_{d-k-2}(\lambda)-r_{k-1}(\lambda) r_{d-k-1}(\lambda) \\
= & {\left[s(\lambda, 1) r_{k-1}(\lambda)-(\lambda-1)^{2} r_{k-2}(\lambda)\right] r_{d-k-2}(\lambda) } \\
& -r_{k-1}(\lambda)\left[s(\lambda, 1) r_{d-k-2}(\lambda)-(\lambda-1)^{2} r_{d-k-3}(\lambda)\right] .
\end{aligned}
$$

Then

$$
\begin{aligned}
& r_{k}(\lambda) r_{d-k-2}(\lambda)-r_{k-1}(\lambda) r_{d-k-1}(\lambda) \\
= & (\lambda-1)^{2}\left[r_{k-1}(\lambda) r_{d-k-3}(\lambda)-r_{k-2}(\lambda) r_{d-k-2}(\lambda)\right] .
\end{aligned}
$$

By a repeated application of this process, we conclude

$$
\begin{aligned}
& r_{k}(\lambda) r_{d-k-2}(\lambda)-r_{k-1}(\lambda) r_{d-k-1}(\lambda) \\
= & (\lambda-1)^{2(k-1)}\left(r_{1}(\lambda) r_{d-2 k-1}(\lambda)-r_{d-2 k}(\lambda)\right) .
\end{aligned}
$$

Therefore

$$
\begin{aligned}
& r_{k}(\lambda) r_{d-k-2}(\lambda)-r_{k-1}(\lambda) r_{d-k-1}(\lambda) \\
= & (\lambda-1)^{2(k-1)}\left[t(\lambda, 1) r_{d-2 k-1}(\lambda)-s(\lambda, 1) r_{d-2 k-1}(\lambda)+(\lambda-1)^{2} r_{d-2 k-2}(\lambda)\right] \\
= & (\lambda-1)^{2(k-1)}\left[(\lambda-1) r_{d-2 k-1}(\lambda)+(\lambda-1)^{2} r_{d-2 k-2}(\lambda)\right] \\
= & (\lambda-1)^{2 k-1}\left[r_{d-2 k-1}(\lambda)+(\lambda-1) r_{d-2 k-2}(\lambda)\right] \\
= & (\lambda-1)^{2 k-1} \varphi_{d-2 k-1}(\lambda) .
\end{aligned}
$$


The last equality being a consequence of (2.2). Replacing in (2.4), we finally get

$$
\phi_{k}(\lambda)-\phi_{k+1}(\lambda)=(a-1) \lambda(\lambda-1)^{2 k-1} \varphi_{d-2 k-1}(\lambda) .
$$

From the Perron-Frobenius Theory for nonnegative matrices [10], if $A$ is a nonnegative irreducible matrix then $\mathrm{A}$ has a unique eigenvalue equal to its spectral radius $\rho(A)$ and $\rho(A)$ increases whenever any entry of $\mathrm{A}$ increases. Hence $\rho(B)<\rho(A)$ if $B$ is a proper submatrix of a nonnegative irreducible matrix $A$.

The next theorem gives a total ordering in $\mathcal{A}_{n, d}$ by the Laplacian index.

Theorem 4. Let $d \geq 4$. Then

$$
\begin{gathered}
\mu_{1}\left(A_{1}\right)=\mu_{1}\left(A_{d-1}\right)<\mu_{1}\left(A_{2}\right)=\mu_{1}\left(A_{d-2}\right)<\ldots<\mu_{1}\left(A_{\left\lfloor\frac{d}{2}\right\rfloor}\right)= \\
\mu_{1}\left(A_{d-\left\lfloor\frac{d}{2}\right\rfloor}\right) .
\end{gathered}
$$

Proof. Since $A_{k}$ and $A_{d-k}$ are isomorphic caterpillars, we may take $1 \leq$ $k \leq\left\lfloor\frac{d}{2}\right\rfloor$. Let $1 \leq k \leq\left\lfloor\frac{d}{2}\right\rfloor-1$. From Corollary $2, \rho\left(M\left(\mathbf{a}_{k}\right)\right)=\mu_{1}\left(A_{k}\right)>1$. Moreover, from the fact that $M\left(\mathbf{a}_{k}\right)$ is a nonnegative irreducible matrix, $\mu_{1}\left(A_{k}\right)$ is a simple eigenvalue. The identity (2.5) involves the polynomials $\phi_{k}(\lambda)$ and $\phi_{k+1}(\lambda)$ of degrees $2 d-2$ which are the characteristic polynomials of $M\left(\mathbf{a}_{k}\right)$ and $M\left(\mathbf{a}_{k+1}\right)$, respectively. Let

$$
\mu_{1}\left(A_{k}\right)=\alpha_{1}>\alpha_{2} \geq \ldots \geq \alpha_{2 d-2}=0
$$

and

$$
\mu_{1}\left(A_{k+1}\right)=\beta_{1}>\beta_{2} \geq \ldots \geq \beta_{2 d-2}=0
$$

be the eigenvalues of $M\left(\mathbf{a}_{k}\right)$ and $M\left(\mathbf{a}_{k+1}\right)$, respectively. Then (2.5) becomes

$$
\lambda \Pi_{j=1}^{2 d-3}\left(\lambda-\alpha_{j}\right)-\lambda \Pi_{j=1}^{2 d-3}\left(\lambda-\beta_{j}\right)=(a-1) \lambda(\lambda-1)^{2 k-1} \varphi_{d-2 k-1}(\lambda) .
$$

We recall that $\varphi_{d-2 k-1}(\lambda)$ of degree $2 d-4 k-2$ is the characteristic polynomial of the matrix $M\left(\mathbf{e}_{d-2 k-1}\right)$ whose spectral radius is $\mu_{1}\left(C\left(\mathbf{e}_{d-2 k-1}\right)\right)$. 
Since $M\left(\mathbf{e}_{d-2 k-1}\right)$ is a proper submatrix of $M\left(\mathbf{a}_{k}\right), \mu_{1}\left(C\left(\mathbf{e}_{d-2 k-1}\right)\right)<$ $\mu_{1}\left(A_{k}\right)$. Hence $\varphi_{d-2 k-1}\left(\mu_{1}\left(A_{k}\right)\right)>0$. We claim $\mu_{1}\left(A_{k}\right)<\mu_{1}\left(A_{k+1}\right)$. Suppose that $\mu_{1}\left(A_{k}\right) \geq \mu_{1}\left(A_{k+1}\right)$. Then $\mu_{1}\left(A_{k}\right) \geq \beta_{j}$ for all $j$. Taking $\lambda=\mu_{1}\left(A_{k}\right)$ in $(2.6)$, we obtain

$$
\begin{gathered}
-\mu_{1}\left(A_{k}\right) \Pi_{j=1}^{2 d-3}\left(\mu_{1}\left(A_{k}\right)-\beta_{j}\right)= \\
(a-1) \mu_{1}\left(A_{k}\right)\left(\mu_{1}\left(A_{k}\right)-1\right)^{2 k-1} \varphi_{d-2 k-1}\left(\mu_{1}\left(A_{k}\right)\right)
\end{gathered}
$$

which is a contradiction because

$$
-\mu_{1}\left(A_{k}\right) \Pi_{j=1}^{2 d-3}\left(\mu_{1}\left(A_{k}\right)-\beta_{j}\right) \leq 0
$$

and

$$
(a-1) \mu_{1}\left(A_{k}\right)\left(\mu_{1}\left(A_{k}\right)-1\right)^{2 k-1} \varphi_{d-2 k-1}\left(\mu_{1}\left(A_{k}\right)\right)>0 .
$$

Therefore $\mu_{1}\left(A_{k}\right)<\mu_{1}\left(A_{k+1}\right)$. This completes the proof.

Theorem 5. Among all complete caterpillars on $n$ vertices and diameter $d$ the largest Laplacian index is attained by $A_{\left\lfloor\frac{d}{2}\right\rfloor}$.

Proof. The case $d=3$ is given in Corollary 1 . If $d \geq 4$, the result follows from Theorem 2 and Theorem 4.

\section{The largest adjacency index among all complete caterpil- lars}

In this section, we find the caterpillar having the largest adjacency index among all complete caterpillars on $n$ vertices and diameter $d$.

Lemma 3. Let $u, v$ be two vertices of a connected graph $G$. For $1 \leq s \leq$ $d(v)$, let $v_{1}, v_{2}, \ldots, v_{s}$ be some vertices in $N_{G}(v)-\left(N_{G}(u) \cup\{u\}\right)$. Let

$$
\mathbf{x}=\left[\begin{array}{lllll}
x_{1} & x_{2} & \ldots & \ldots & x_{n}
\end{array}\right]^{T}
$$

be the unit Perron vector of $G$ corresponding to the adjacency index $\lambda_{1}(G)$. Let

$$
G_{u}=G-v v_{1}-\ldots-v v_{s}+u v_{1}+\ldots+u v_{s} .
$$

If $x_{u} \geq x_{v}$ then $\lambda_{1}\left(G_{u}\right)>\lambda_{1}(G)$. 
Proof. By hypothesis, $x_{u} \geq x_{v}$. Then

$$
\begin{aligned}
\lambda_{1}\left(G_{u}\right)-\lambda_{1}(G) & \geq \mathbf{x}^{T} A\left(G_{u}\right) \mathbf{x}-\mathbf{x}^{T} A(G) \mathbf{x} \\
& =2\left(x_{u}-x_{v}\right) \sum_{i=1}^{s} x_{i} \geq 0 .
\end{aligned}
$$

Suppose that $\lambda_{1}\left(G_{u}\right)=\lambda_{1}(G)$. Then, from the above inequality, we get

$$
\mathbf{x}^{T} A\left(G_{u}\right) \mathbf{x}=\mathbf{x}^{T} A(G) \mathbf{x}=\lambda_{1}(G)=\lambda_{1}\left(G_{u}\right) .
$$

Since $A\left(G_{u}\right)$ is a real symmetric matrix, from $\mathbf{x}^{T} A\left(G_{u}\right) \mathbf{x}=\lambda_{1}\left(G_{u}\right)$, we obtain

$$
A\left(G_{u}\right) \mathbf{x}=\lambda_{1}\left(G_{u}\right) \mathbf{x}
$$

It follows that

$$
\lambda_{1}\left(G_{u}\right) x_{v}=\sum_{w \in N_{G_{u}}(v)} x_{w}
$$

Moreover

$$
\lambda_{1}(G) x_{v}=\sum_{w \in N_{G}(v)} x_{w}=\sum_{w \in N_{G_{u}}(v)} x_{w}+\sum_{i=1}^{s} x_{v_{i}} .
$$

Subtracting (3.1) from (3.2), we obtain

$$
0=\sum_{i=1}^{s} x_{v_{i}}>0
$$

which is a contradiction. Hence $\lambda_{1}\left(G_{u}\right)>\lambda_{1}(G)$.

We comment that a version of Lemma 3 for the Laplacian index of a connected bipartite graph is given in [4].

An immediate consequence of Lemma 3 is

Lemma 4. Let $u, v$ be two vertices of a connected graph $G$. For $1 \leq s \leq$ $d(v)$, let $v_{1}, v_{2}, \ldots, v_{s}$ be some vertices in $N_{G}(v)-\left(N_{G}(u) \cup\{u\}\right)$. For $1 \leq t \leq d(u)$, let $u_{1}, u_{2}, \ldots, u_{t}$ be some vertices in $N_{G}(u)-\left(N_{G}(v) \cup\{v\}\right)$.

Let

$$
G_{u}=G-v v_{1}-v v_{2}-\cdots-v v_{s}+u v_{1}+u v_{2}+\cdots+u v_{s}
$$

and

$$
G_{v}=G-u u_{1}-u u_{2}-\cdots-u u_{t}+v u_{1}+v u_{2}+\cdots+v u_{t} .
$$

Then $\lambda_{1}\left(G_{u}\right)>\lambda_{1}(G)$ or $\lambda_{1}\left(G_{v}\right)>\lambda_{1}(G)$. 
By a repeated application of Lemma 4, using a similar argument to the proof of Theorem 2, we obtain

Theorem 6. Let $d \geq 3$. Let $C(\mathbf{p}) \in \mathcal{C}_{n, d}$ with $\mathbf{p}=\left[p_{1}, \ldots, p_{d-1}\right]$. There exists a caterpillar $A_{k} \in \mathcal{A}_{n, d}$ for some $1 \leq k \leq d-1$ such that $\lambda_{1}\left(A_{k}\right) \geq$ $\lambda_{1}(C(\mathbf{p}))$.

Corollary 4. If $d=3$ then $C(n-3,1)$ has the largest adjacency index among all trees on $n$ vertices and diameter 3 .

Proof. Clearly $A_{1}=C(n-3,1)$ and $A_{2}=C(1, n-3)$ are isomorphic caterpillars. Since any tree of diameter 3 is a complete caterpillar, from Theorem $6, \lambda_{1}\left(A_{1}\right)=\lambda_{1}\left(A_{2}\right) \geq \lambda_{1}(T)$ for any tree $T$ on $n$ vertices and diameter 3 .

Now, we order the caterpillars in $\mathcal{A}_{n, d}$ by their adjacency indices. From Theorem 6 in [7], we have

Theorem 7. The adjacency eigenvalues of $C(\mathbf{p})$ are 0 with multiplicity $\sum_{i=1}^{d-1} p_{i}-(d-1)$ and the eigenvalues of the $(2 d-2) \times(2 d-2)$ irreducible nonnegative matrix

$$
H(\mathbf{p})=\left[\begin{array}{ccccc}
S\left(p_{1}\right) & E & & & \\
E & S\left(p_{2}\right) & E & & \\
& \ddots & \ddots & \ddots & \\
& & \ddots & S\left(p_{d-2}\right) & E \\
& & & E & S\left(p_{d-1}\right)
\end{array}\right]
$$

where

$$
S(x)=\left[\begin{array}{cc}
0 & \sqrt{x} \\
\sqrt{x} & 0
\end{array}\right], E=\left[\begin{array}{ll}
0 & 0 \\
0 & 1
\end{array}\right]
$$

An immediate consequence of Theorem 3 is

Corollary 5. The spectral radius of $H(\mathbf{p})$ is the adjacency index of $C(\mathbf{p})$.

Let $s(\lambda, x)$ be the characteristic polynomial of $S(x)$. That is

$$
s(\lambda, x)=\lambda^{2}-x .
$$

We now apply Lemma 2 to the matrix $H(\mathbf{p})$. 
Corollary 6. The characteristic polynomial of $H(\mathbf{p})$ is

$$
=\left|\begin{array}{ccccc}
s\left(\lambda, p_{1}\right) & -\lambda & & & \\
-\lambda & s\left(\lambda, p_{2}\right) & -\lambda & & \\
& \ddots & \ddots & \ddots & \\
& & \ddots & s\left(\lambda, p_{d-2}\right) & -\lambda \\
& & & -\lambda & s\left(\lambda, p_{d-1}\right)
\end{array}\right|_{d-1} .
$$

We have $A_{k}=C\left(\mathbf{a}_{k}\right)$. Since the adjacency index of $C(\mathbf{p}) \in \mathcal{C}_{n, d}$ is equal to the spectral radius of $H(\mathbf{p})$, to order the caterpillars in $\mathcal{A}_{n, d}$ by their adjacency indices is equivalent to order the matrices

$H\left(\mathbf{a}_{1}\right), H\left(\mathbf{a}_{2}\right), \ldots, H\left(\mathbf{a}_{d-1}\right)$ by their spectral radii. We may take $1 \leq k \leq$ $\left\lfloor\frac{d}{2}\right\rfloor$.

Let

$$
\phi_{k}(\lambda)=\left|\lambda I-H\left(\mathbf{a}_{k}\right)\right|
$$

Let

$$
r_{0}(\lambda)=1, r_{1}(\lambda)=s(\lambda, 1)
$$

and, for $2 \leq k \leq\left\lfloor\frac{d}{2}\right\rfloor$, let

$$
r_{k}(\lambda)=\left|\begin{array}{ccccc}
s(\lambda, 1) & -\lambda & & & \\
-\lambda & \ddots & -\lambda & & \\
& \ddots & \ddots & \ddots & \\
& & \ddots & s(\lambda, 1) & -\lambda \\
& & & -\lambda & s(\lambda, 1)
\end{array}\right|_{k}
$$

Expanding along the first row, we have

$$
r_{k}(\lambda)=s(\lambda, 1) r_{k-1}(\lambda)-\lambda^{2} r_{k-2}(\lambda)
$$

Clearly $s(\lambda, a)=s(\lambda, 1)+(1-a)$. Let $1 \leq k \leq\left\lfloor\frac{d}{2}\right\rfloor-1$.

Applying the same techniques of Section 2, the difference $\phi_{k}(\lambda)-$ $\phi_{k+1}(\lambda)$ becomes

$$
\phi_{k}(\lambda)-\phi_{k+1}(\lambda)=(a-1) \lambda^{2 k} r_{d-2 k-2}(\lambda) .
$$

The next theorem gives a total ordering in $\mathcal{A}_{n, d}$ by the adjacency index. 
Theorem 8. Let $d \geq 4$. Then

$\lambda_{1}\left(A_{1}\right)=\lambda_{1}\left(A_{d-1}\right)<\lambda_{1}\left(A_{2}\right)=\lambda_{1}\left(A_{d-2}\right)<\ldots<\lambda_{1}\left(A_{\left\lfloor\frac{d}{2}\right\rfloor}\right)=\lambda_{1}\left(A_{d-\left\lfloor\frac{d}{2}\right\rfloor}\right)$.

Proof. Similar to the proof of Theorem 4.

Theorem 9. Among all complete caterpillars on $n$ vertices and diameter $d$ the largest adjacency index is attained by $A_{\left\lfloor\frac{d}{2}\right\rfloor}$.

Proof. The case $d=3$ is given in Corollary 4. If $d \geq 4$, the result follows from Theorem 6 and Theorem 8 .

\section{References}

[1] D. Cvetković, P. Rowlinson, S. K. Simić, An Introduction to the Theory of Graph Spectra, London Mathematical Society, Student Texts 75, Cambridge University Press, (2010).

[2] J.M. Guo, J-Y. Shao, On the spectral radius of trees with fixed diameter, Linear Algebra and its Applications 413, pp. 131-147, (2006).

[3] J.- M. Guo, On the Laplacian spectral radius of trees with fixed diameter, Linear Algebra Appl. 419, pp. 618-629, (2006).

[4] J.- M. Guo, The effect on the Laplacian spectral radius of a graph by adding or grafting edges, Linear Algebra Appl. 413, pp. 59-71, (2006).

[5] O. Rojo, L. Medina, N. Abreu and C. Justel, On the algebraic connectivity of some caterpillars: A sharp upper bound and a total ordering. Linear Algebra and its Applications 432, pp. 586-605, (2010).

[6] O. Rojo, L. Medina, N. Abreu and C. Justel, Extremal algebraic connectivities of certain caterpillar classes and symmetric caterpillars, Electronic Journal of Linear Algebra, Vol. 20, pp. 136-157, (2010).

[7] O. Rojo, L. Medina, Spectra of generalized Bethe trees attached to a path, Linear Algebra and its Applications 430, pp. 483-503, (2009). 
[8] O. Rojo, L. Medina, Spectra of weighted compound graphs of generalized Bethe trees, Electronic Journal of Linear Algebra 18, pp. 30-57, (2009).

[9] S. K. Simić, E. M. L. Marzi, F. Belardo, On the index of caterpillars, Discrete Mathematics 308, pp. 324-330, (2008).

[10] R. S. Varga, Matrix Iterative Analysis, Springer Series in Computational Mathematics, Volume 27, (2000).

\section{Nair Abreu}

Production Engineering Program, PEP/COPPE

Universidade Federal do Rio de Janeiro

Rio de Janeiro,

Brazil

e-mail: nairabreunovoa@gmail.com

\section{Eber Lenes}

Departamento de Investigaciones

Universidad del Sinú. Elías Bechara Zainúm

Cartagena,

Colombia

e-mail:elenes@ucn.cl

and

\section{O. Rojo}

Department of Mathematics

Universidad Católica del Norte

Antofagasta,

Chile

e-mail : orojo@ucn.cl 